\title{
TERRESTRIAL SCANNING OR DIGITAL IMAGES IN INVENTORY OF MONUMENTAL OBJECTS? - CASE STUDY
}

\author{
J.S. Markiewicz ${ }^{a^{*}}$, D. Zawieska ${ }^{a}$ \\ ${ }^{a}$ Faculty of Geodesy and Cartography, Institute of Photogrammetry, Remote Sensing and Spatial Information Systems, Warsaw \\ University of Technology, Warsaw, Poland - (j.markiewicz, d.zawieska)@ gik.pw.edu.pl
}

\author{
Commission VI, WG V/2
}

KEY WORDS: Terrestrial scanning, dense image matching, monumental objects, orthoimages

\begin{abstract}
:
Cultural heritage is the evidence of the past; monumental objects create the important part of the cultural heritage. Selection of a method to be applied depends on many factors, which include: the objectives of inventory, the object's volume, sumptuousness of architectural design, accessibility to the object, required terms and accuracy of works. The paper presents research and experimental works, which have been performed in the course of development of architectural documentation of elements of the external facades and interiors of the Wilanów Palace Museum in Warszawa. Point clouds, acquired from terrestrial laser scanning (Z+F 5003h) and digital images taken with Nikon D3X and Hasselblad H4D cameras were used. Advantages and disadvantages of utilisation of these technologies of measurements have been analysed with consideration of the influence of the structure and reflectance of investigated monumental surfaces on the quality of generation of photogrammetric products. The geometric quality of surfaces obtained from terrestrial laser scanning data and from point clouds resulting from digital images, have been compared.
\end{abstract}

\section{INTRODUCTION}

Generation of precise and, at the same time, high resolution architectural documentation, acquired on the basis of terrestrial laser scanning or dense point clouds from digital images is still and open issue, which forms many challenges (Boehle, 2004; Remondino, 2006, Remonidno 2008; Droninger, 2013; Mousa, 2014) . Although data processing algorithms are intensively developed with consideration of high resolution orthoimage processing or generation of accurate vector drawings, limitations concerning the utilisation of measuring sensors are still the issue which should be solved (Boehler, 2004). In the case of processing data acquired from terrestrial laser scanning existing issues are related to projection of objects, which are connected with the cultural heritage, in its wide sense.

Many issues can be dealt with in the process of processing of such objects. One of those issues, which relatively often occurs is the lack of possibility to fully visualise the entire object using one data source (one measuring sensor) (Markiewicz 2012). Such limitations may result from the lack of access or from invasive operations of the measuring system. Another issue is related to the complicated geometry, as well as the structure and texture of investigated surfaces. An alternative for such limitations seems to be utilisation of many sensors, mainly terrestrial laser scanning, and digital images (Angello, 2007). Such systems are often complementary, in particular, in the cases of measurements of complicated, monumental surfaces.

\section{METHODOLOGY}

\subsection{Terrestrial Laser Scanning}

Terrestrial laser scanning is a $3 \mathrm{D}$ measurement technique which is based on a laser beam deflection. This kind of survey allows to rapidly acquire a lot of information with a high degree of detail. Each measured point contains coordinates and radiometric information (reflectance and/or RGB values). Laser scanning systems can produce data that can vary in terms of point density, field-of-view (FOV), amount of noise, incident angle, waveform and texture information (Grussenmeyer, 2012). The main advantage of this technique is a possibility of measurement of low quality or even un-textured areas. In spite of fact that it has a lot of advantages, there are several problems which have influence on the acquisition accuracy (Lerma, 2008), e.g.:

- Laser beam propagation.

- Mixed edge problem it is one of the most important consequences of the beam divergence and it occurs when the laser beam hits an edge of an object .It is divided into the two parts: one part reflects and the other one travels further and hits another surface. This unwanted behaviour generates noise, because the coordinates of this point are calculated as an average of several returned signals.

- Object reflection errors: This kind of errors are the result of the reflectance. For very dark materials surfaces absorb most of the visible spectrum, so the reflectance signal is very weak Therefore the point accuracy will be corrupted by the noise. On the other hand surfaces with then high reflectance (i.e. bright surfaces) give more reliable and precise range measurement. However, if the object reflectivity is too high (metal surface, retro reflective tape etc.), the laser beam is fully deflected in the mirroring direction, which leads to improper measurements.

- Temperature and atmosphere 


\section{2.}

\section{Digital Photogrammetry}

Nowadays, Close-range Photogrammetry (image-based) depends on SfM ('Structure form Motion') approach which is a combination of photogrammetric and computer vision methods. It is a fully automated $3 \mathrm{D}$ reconstruction technique, which refers to simultaneous estimation of camera orientation, self-calibration and dense point cloud generation. (Mousa, 2014). Image-based approach of an object reconstruction is generally establishing correspondence between primitives extracted from minimum two, but usually more images. Those corresponding primitives are converted into the $3 \mathrm{D}$ point cloud using the mathematical model. The main aspect connected with the image-based method is the possibility of the image correlation is the most important issue during the $3 \mathrm{D}$ reconstruction. This problem is studied since more than 30 years but still many problems exist such as: no possibility of completeness automation, occlusions, poor or un-textured areas, repetitive structures, etc. (Remondino 2008). In order to reduce mentioned problems and generate dense and accurate point cloud, the camera's stations should be appropriately distributed. During the photogrammetric network design, a set of rules on how to collect images, where to set up a camera positions or how many images to capture. should be fulfilled Those factors are (Fraser, 1996; Clarke, 1998; El-Hakim, 2003; Mousa, 2014):

- The ratio base-to-depth (b/d): the network accuracy increase with the increase of this ratio and using convergent images rather than images with parallel optical axes.

- The number of captured images: the accuracy improves significantly with the number of images where a point appears. But measuring the point in more than four images gives less significant improvement.

- The number of the measured point in each images: the accuracy increase with the number of measured point per images. However the increase is not significant if the geometric configuration is strong and the points being measured are well defined (like targets) and well distributed in the image. In addition, this also applies for the utilized control points.

- The image resolution (number of pixels): on natural features, the accuracy improves significantly with the image resolution, while the improvement is less significant on well-defined largely resolved targets.

\section{SPECIFICATIONS OF MONUMENTAL OBJECTS AND PERFORMED MEASUREMENTS}

The paper presents tests and experiments which were performed in the process of developing architectural documentation of elements of the external facade (Fig.1) and interiors of the Museum of king Jan III's Palace at Wilanów in Warsaw, Poland (Fig.2).

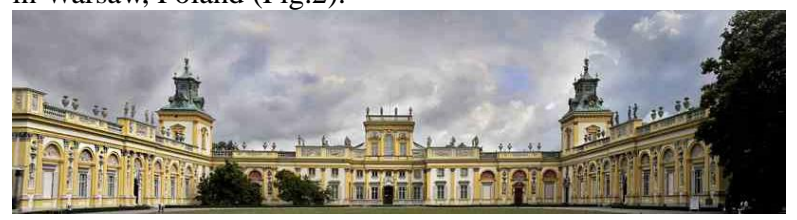

Figure. 1 Wilanów Palace, Warsaw, Poland (source: http://www.wilanow-palac.pl/)

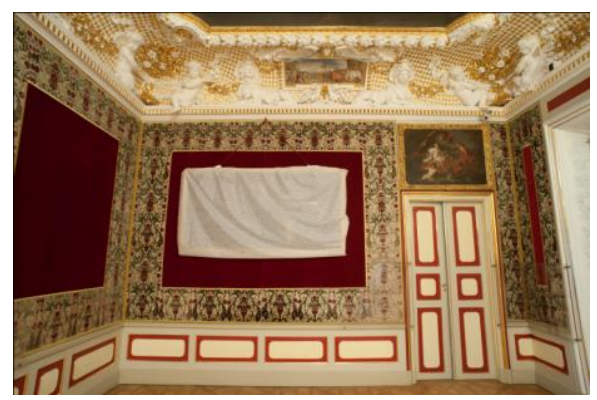

Figure 2. A fragment of the historical sleeping room of the Queen at the Wilanów Palace (Warsaw, Poland)

Point clouds acquired from terrestrial laser scanning $(\mathrm{Z}+\mathrm{F}$ 5003 scanner) and digital images (Nikon D3X, Hasselblad H4D cameras) were utilised. Images of facades were taken in the natural illumination (slight cloudiness); photographs of interiors were taken in the mixed illumination conditions, i.e. with the use of flash lamps and the existing light. Flash lamps were located in order to equalise the artificial light, and not to dominate it. Illumination was scattered by softboxes and reflected; not direct illumination was applied.

\section{COMPARISON OF TLS AND AUTOMATIC IMAGE MATCHING TECHNOLOGIES}

Sessions of measurements of historical external facades and interiors of the Palace were performed in various periods. Some of architectural details (sculptures) were restored at that periods. Due to this some of the details and sculptures were not measured at the time of laser scanning and they were projected later on photographs. That is why it was necessary to integrate resulting products acquired from both data sources (Fig. 3). For the data processing Lopscan and Agisoft software were used.

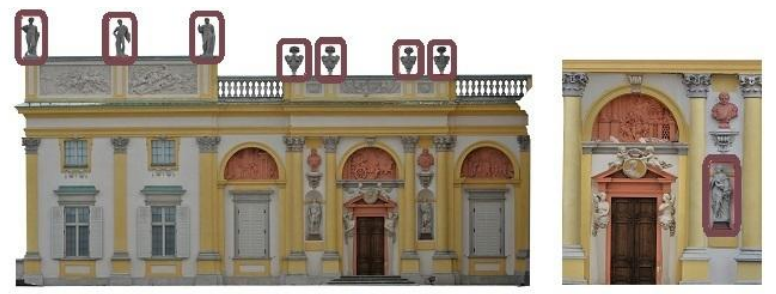

Figure 3 Fragments of orthoimages of the historical facade from TLS with marked elements acquired from the image matching process.

In the course of processing the cloud of points from terrestrial laser scanning it turned out, that due to the complicated geometry of the object and the presence of many adornments, gilded details and bas-reliefs were projected with errors and it was necessary to correct this data (Fig.4). Dense point clouds were used for that purpose, which were generated from digital image matching. Thus, geometric positions of TLS data were corrected and missing data (blind spots) were added.

The additional factor which influence the need to utilise the point cloud from digital images was the lack of data in data sets from terrestrial laser scanning; this resulted from the presence of dense vegetation, which covered fragments of facades of the objects (Fig. 1).

During generation of high resolution orthoimages of historical interiors of the Museum of king Jan III's Palace at Wilanów it was also analysed, how the reflectance and structure of walls, covered with ornamental fabrics, with 

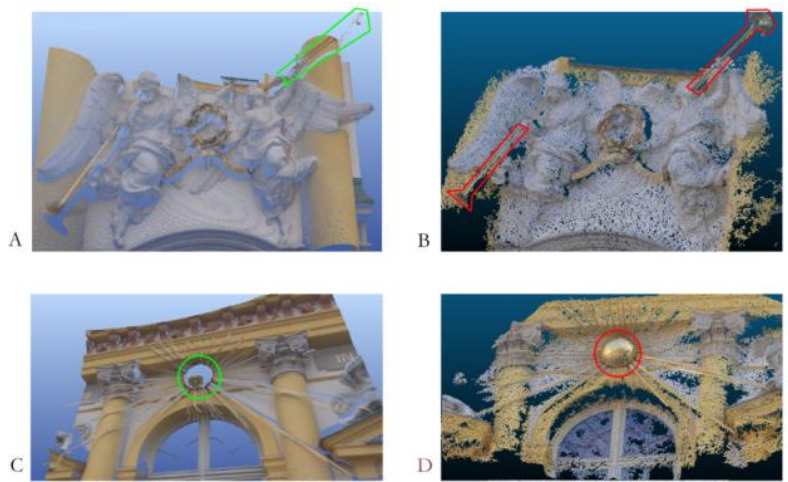

Figure 4 A, C - Clouds of points of gilded details, acquired from TLS. B, D - clouds of points acquired from digital image matching (red - blind spots, green - geometric corrections).

many gilded details, influence the quality of photogrammetric products.

The geometric quality of surfaces obtained from laser scanning was compared with their quality obtained from clouds of points generated from digital images (Fig.5).

In order to check the accuracy of projection of historical fabrics (Fig.. 5 IA and IC) deviations of points from the approximated reference plane (a fragment of a wall covered with fabrics) were analysed. In the case of a fragment projected on the basis of data from terrestrial laser scanning the mean distance between the points and the plane was equal to $21 \mathrm{~mm} ; 31 \%$ of points projected $\pm 1-2 \mathrm{~mm}, 40 \%$ about $\pm 2-3 \mathrm{~mm}$ and $29 \%$ less than $1 \mathrm{~mm}$, as a $\mathrm{n}$ average. In the case of point clouds resulting from image matching, the average deviation equalled to $1.5 \mathrm{~mm}$, including $21 \%$ of points which projected about $\pm 1-2 \mathrm{~mm}, 18 \%$ about $\pm 2-3 \mathrm{~mm}$ and $61 \%$ - less than $1 \mathrm{~mm}$, as an average.

As it turns out from Fig.6A, the highest deviations of point clouds resulted from image matching and they occur in such places where the explicit texture is missing; in the case of decorations deviations greater than $1 \mathrm{~mm}$ were not observed. As it turns out from Fig 6B, great deviations occur on gilded surfaces; for flat elements deviation greater than $1 \mathrm{~mm}$ were not observed. Scanner measurements and photographs were acquired in similar illumination conditions, and the scanner stations was located in the centre of a room, what resulted in the laser beam incidence angle value, which was not too acute.

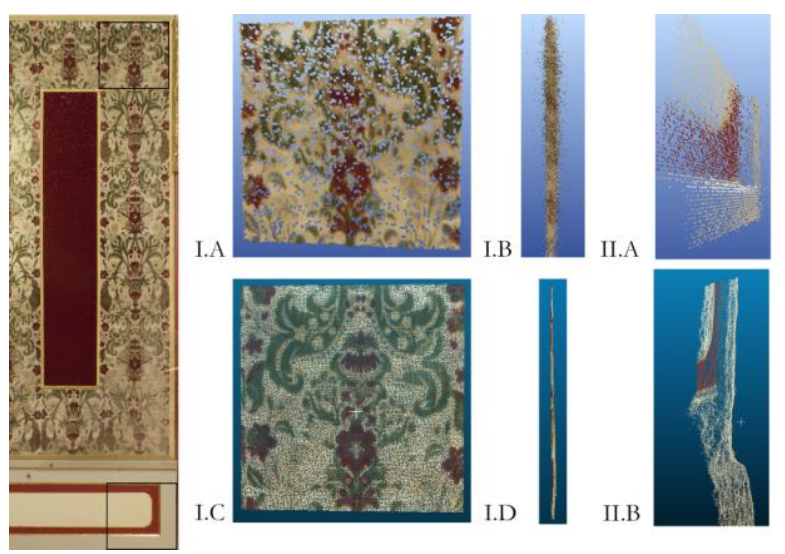

Figure 5 Cloud of points: I. ornamental fabrics: I.A orthogonal projection, parallel to the plane (TLS), I.Borthogonal projection, perpendicular to the plane (TLS), I.C orthogonal projection, parallel to the plane (Matching), I.Dorthogonal projection, perpendicular to the plane (Matching);

II. Plaster: II.A axonometric projection (TLS), II.Baxonometric projection (Matching)

A fragment of walls covered with plaster and oil paint was also tested, together with decorated, gilded roundings.

Fig. 6 presents deviations from theoretical shapes presented in Fig. 5 IIA and 5 IIB.

In the case of point clouds resulting from image matching, the average deviation equalled to $6 \mathrm{~mm} ; 34 \%$ of points which projected about $\pm 1-2 \mathrm{~mm}, 32 \%$ - about $\pm 2-3 \mathrm{~mm}$ and $34 \%$ - less than $1 \mathrm{~mm}$, as an average. In the case of a fragment projected on the basis of data from terrestrial laser scanning, the mean distance between points and the plane was equal to $1.7 \mathrm{~mm} ; 33 \%$ of points projected about $\pm 1-2 \mathrm{~mm}, 35 \%-$ about $\pm 2-3 \mathrm{~mm}$ and $32 \%$ - less than $1 \mathrm{~mm}$.

It may be seen from performed analyses that it seem useful to combine two data acquisition techniques. In such a case it would be possible to eliminate erroneous points from data sets, without the danger of creation of blind spots
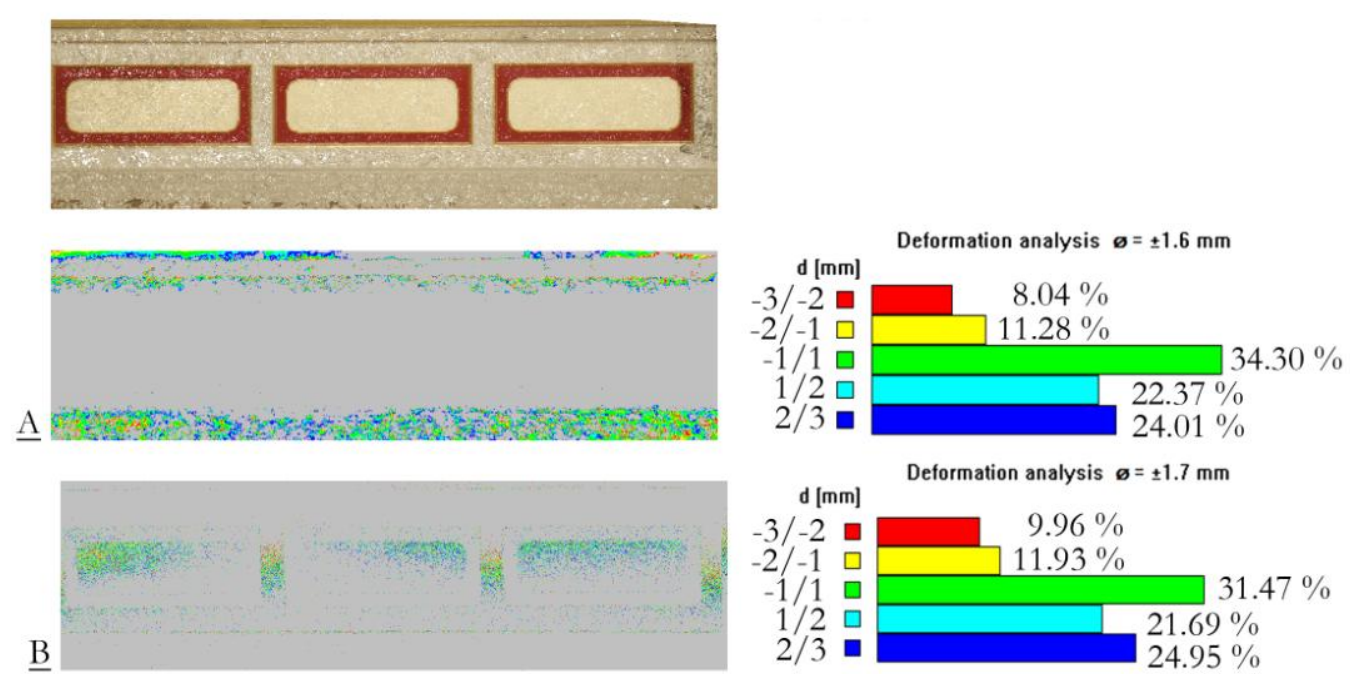

Figure 6 Deviations of points from reference planes. A - the point cloud from image matching, B - the point cloud from terrestrial laser scanning. The null deviations are presented in grey. 
$\underline{\mathrm{A}}$
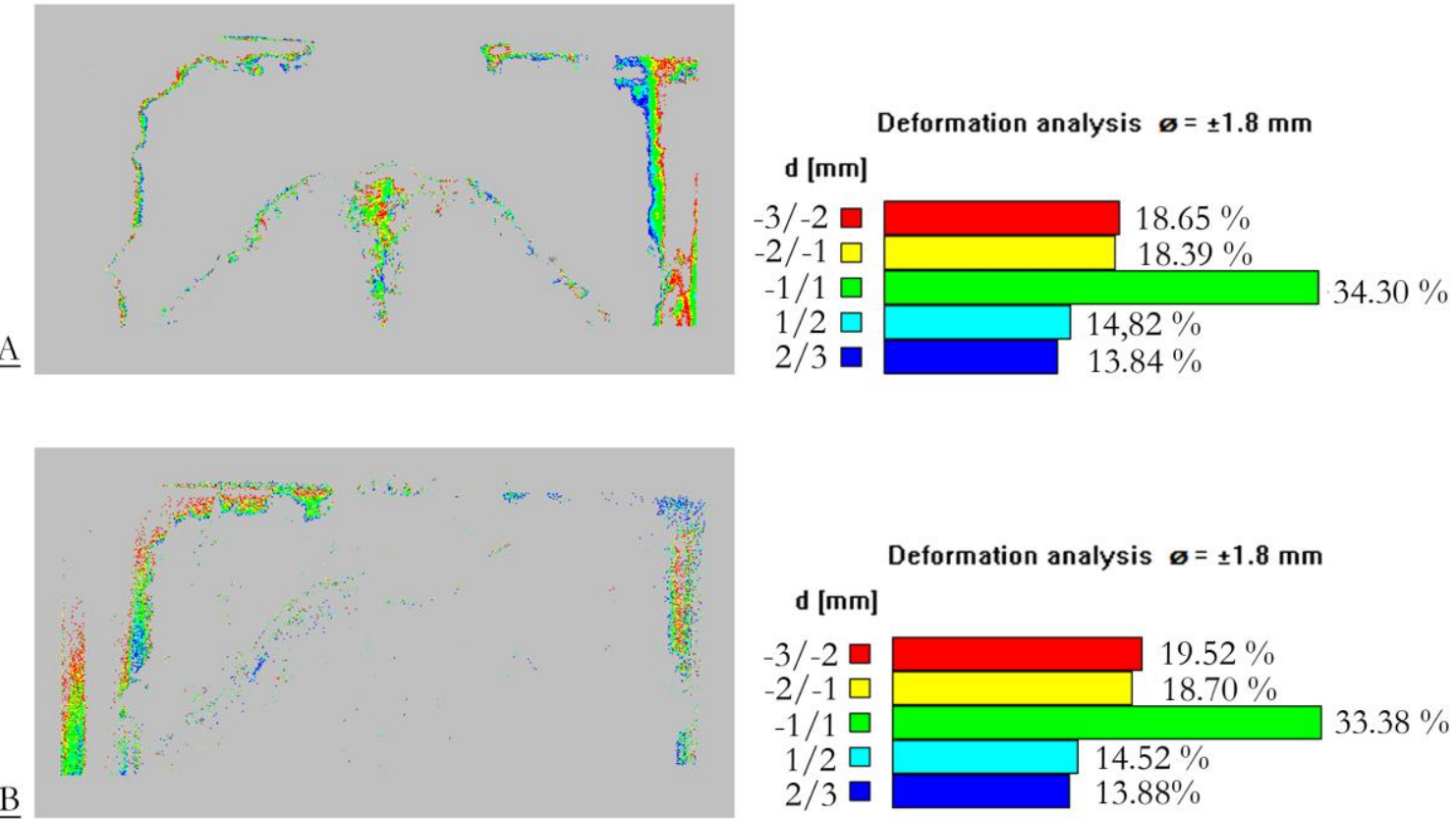

Figure. 7. A Deviations of points from reference planes. A - the point cloud from image matching, B - the point cloud from terrestrial laser scanning. The null deviations are marked in grey.

Integration of both products allowed for geometric correction of laser scanning data, with errors resulting from erroneous determination of positions of points on gilded details (Fig. 6) and on ornamental fabrics.

The analysis also covered point clouds acquired for gilded decorations of complicated geometric features (Fig. $8 \mathrm{~A}$ and D). Results of analyses are presented in Fig. 7. As a result of utilisation of artificial light it was possible to eliminate reflexes; as a result the texture was evenly illuminated. However, in the case of too short distances in rooms, scanned elements and gildings are projected with the lower intensity of reflection. However, in the case of the natural illumination, outside the building, measurements of decorations may result not only in the small number of points, but also in the lack of data (Fig. $4 \mathrm{~A}$ and C). This is often caused by disadvantageous atmospheric conditions, as well as longer distances and the structure and reflectance of the object.
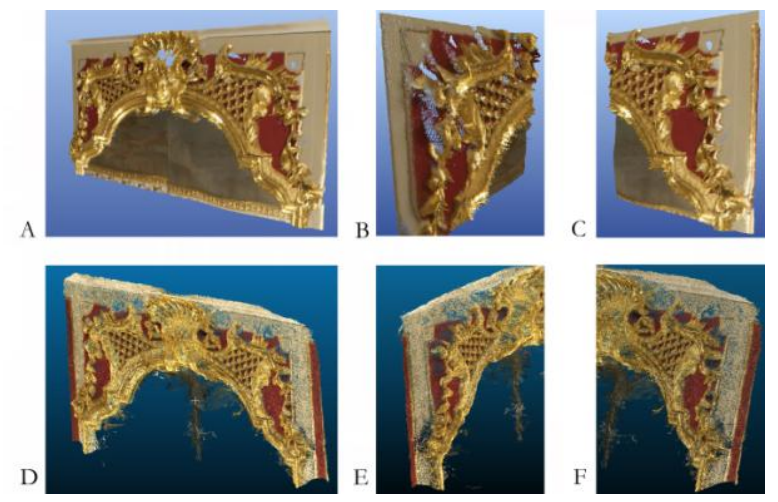

Figure. 8 Cloud of points of gilded details TLS (A, B, C various views) and image matching (D, E , F - various views)

Similarly to previous analyses, deviations between the reference planes and point clouds for both data sources were also tested for this element (Fig. 8). It may be noticed that distribution of deviations is smaller in the case of data resulting from automatic image matching than in the case of the laser scanning data. Another advantage results from the fact that these deviations are systematically distributed and they are probably caused by the worse configuration of photographs of the right-sided decorations. It also turns from the Fig.7 that deviations between points acquired from both method are comparable.

Another task which was to be solved, concerned the processing of surfaces of caissons, which connect walls with ceilings; they were gilded, with many details and their shape was close to the shape of a roller. TLS data is presented in Fig. 9A, 9C, where many blind spots are visible, as well as deformed details, resulting from the acute angle of incidence of the laser beam, mutual overlapping of details and reflective surfaces.
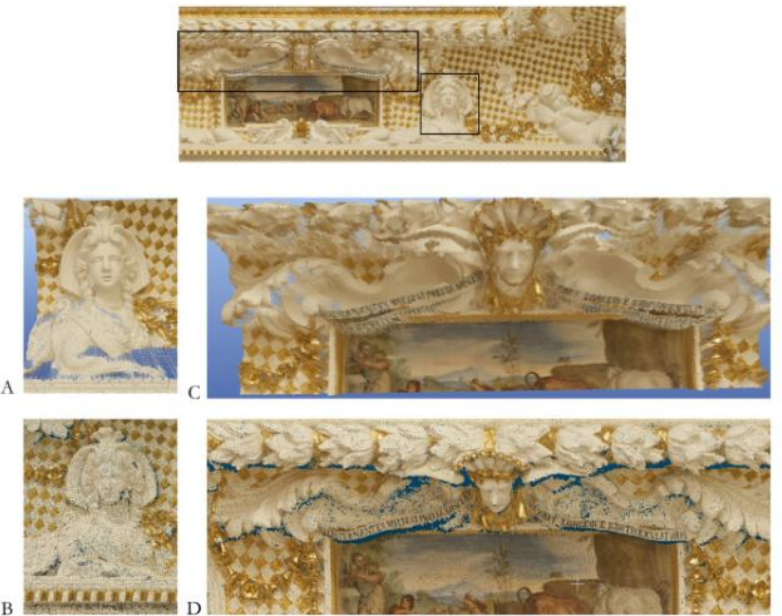

Figure. 9 Selected clouds of points of gilded details of caissons A, C-TLS , B, D - from image matching

Unfortunately, this is caused not only by the specific features of the object itself, but also by the accessibility of all details. In the case of the discussed object it was not possible to utilise scaffolds, since the room was excluded from the everyday use for a short time only. 
$\underline{A}$

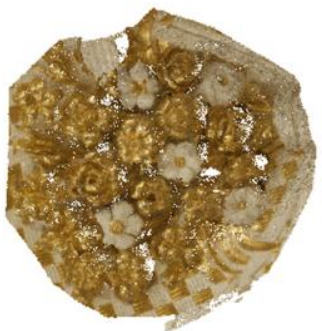

$\underline{\mathrm{C}}$

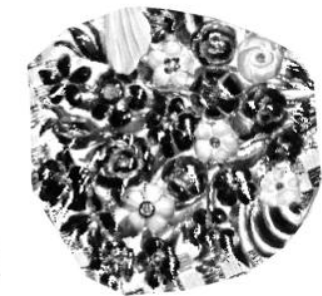

$\underline{B}$
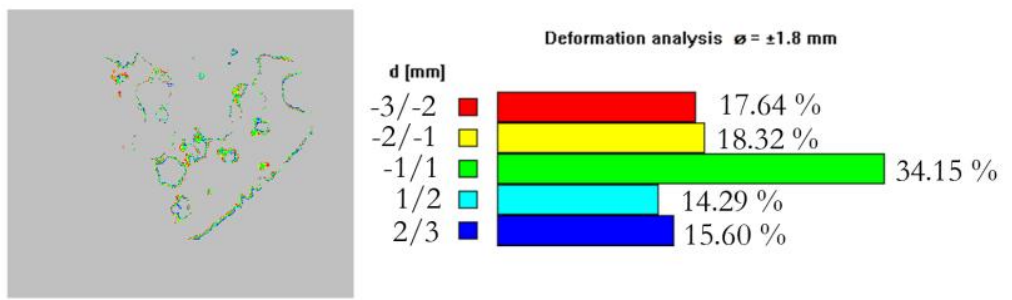

$\underline{\mathrm{D}}$
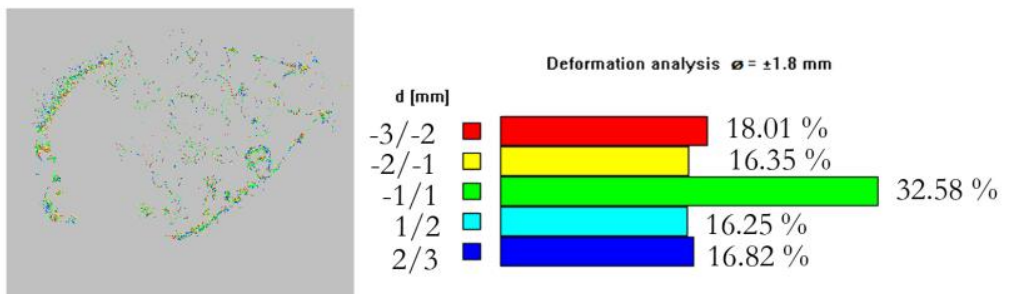

Fig. 10 Orthimage of analyzed detail. A- from image matching, C - from terrestrial laser scanning. Deviations of points from reference planes. B - the point cloud from image matching, D - the point cloud from terrestrial laser scanning.

The null deviations are marked in grey.

The additional limitation which influenced the possibility to project the details, was the size and weight of the scanner, which did not allow for placing it in an arbitrary place. Limitations in utilisation of the scanner were caused by the distance from the scanned elements was too short; therefore gilded elements were projected with the lower intensity of reflection and the lower accuracy of point positioning. The effect of duplication of points may be noticed in Fig. 9C. The same illumination conditions, the level of the scanner and camera stations and the perpendicular direction of acquisition of photographs and scanning were assumed in the performed experiments. Fig.10 presents the analysis of projection of a complicated shape of a detail, located on the ceiling. It results, that deviations for the point clouds are similar with respect to numerical values. The explicit trend of their distribution may be noticed. In the case of point clouds, acquired from image matching, they are characterised by the low dispersion, which occurs at the edges of decorated flowers only. In the case of point clouds acquired from TLS, noises are visible, which may not be explicitly eliminated from the point cloud. They are the results of the laser beam deflection.

When analysing the experiments performed it may be concluded that improvement of the geometry of the projected shape of a detail, as well as complementing the missing data is possible due to utilisation of point clouds, acquired from the dense image matching.

\section{CONCLUDING REMARKS}

In the course of measurements of monumental objects many difficulties may be met, which are the result of the object specific features, as well as limitations of utilised sensors. When objects located outside are processed, the time and existing weather conditions should be considered. When laser scanning is the only applied technology, this consideration may be neglected due to the specific features of measurements. In the case when photographs are to be applied (not only for the needs of colouring the point cloud), it is necessary to acquired photographs in cloudy conditions. Unfortunately, this is not always possible. These difficulties may be solved by, for example, utilisation of the HDR technique, which may be applied with the sue of a camera dedicated for the scanner Z+F 5010. Thanks to the HDR complicated geometric features of details and decorations, resulting in noises and erroneous projection of points. Unfortunately, in the course of measurements of details with the use of a phase scanner $\mathrm{Z}+\mathrm{F} 5003 \mathrm{~h}$, in historical rooms, technique (Ntegeka 2013, ZF, 2014) it is possible to eliminate unexposed and overexposed fragments, as a result of changing the values of exposition parameters. Thanks to the utilisation of the existing illumination it is possible to acquire photographs in the natural and correct colours. On the other hand, utilisation of professional illumination allows for elimination of penumbras, tone differences, and dark, unexposed elements. Utilisation of the HDR technique would be useful for taking photographs outside in the field; however, in the case of historical rooms, it turns out from the experiences gained by the authors, that it is recommended to apply the professional illumination.

Basing on the performed experiments and research works, several practical comments may be made, which may be utilised when monumental objects are measured with the use of TLS and matching of blocks of digital images:

- terrestrial laser scanning should be applied for processing historical objects or their parts, which do not have a clear and explicit texture;

- the utilisation of the scanner is limited not only by the way and method of measurements, but also by its size, weight and the lack of possibility to locate the scanner in an arbitrary place. Utilisation of a relevant geometry of a block of terrestrial images may be an alternative solution;

- utilisation of a specially planned block of terrestrial photographs, acquired in relevant illumination conditions allows for obtaining high accuracy of results. It is important to avoid reflexes;

- gilded decorations should be processed with the use of data from the dense matching, since positioning errors from terrestrial laser scanning are not systematic and they cannot be eliminated in an explicit way;

- when fabrics, decorative tapestries are processed, point clouds from dense image matching should be acquired, since utilisation of the TLS projects surfaces with errors and the effect of noise occurs.

To sum up it may be stated that in the course of selection of the technology of measurements of monumental objects, not only the possibility to access the object or the speed of measurements should be initially evaluated; the type of the 
structure and the reflectance of a measured surface and illumination conditions should be also considered. All of those factors influence the accuracy of generated photogrammetric products. In many cases, it is useful to apply both technologies, which are often complementary to one another.

\section{REFERENCES}

Agnello, F., Lo Brutto, M. 2007. Integrated surveying techniques in cultural heritage documentation. In: The International Archives of the Photogrammetry, Remote Sensing and Spatial Information Sciences, Zurich, Switzerland, Vol. XXXVI, Part TS 3.

Boehler W., Marbs A., 2004. 3D scanning and photogrammetry for heritage recording: a comparison. Proceedings 12th Int. Conf. on Geoinformatics -Geospatial Information Research: Bridging the Pacific and Atlantic, Gävle, Sweden, pp. 291-298.

Clarke T. A., Wang X., Fryer J.G., 1998. The principal point and CCD. ThelPhotogrammetry Record, Volume 16, pp. $293-312$

Dorninger P., Nothegger C., Rasztovits S., 2013. Efficient 3D documentation of Neptune fountain in the park of Schönbrunn palace at millimetre scale. In: ISPRS Annals of the Photogrammetry, Remote Sensing and Spatial Information Sciences, Strasbourg, France Volume II-5/W1, pp.103 - 108 .

El - Hakim S.F., Berladin J.A., Blais F., 2003. Critical factors and configurations for practical 3D image-based modeling. In: VI Conference on Optical 3D Measurement Techniques, Zurich, Switzerland, pp. 159 - 167

Fraser C.S., 1996 Network design. Photogrammetry and Machine Vision. Whittles Pub, pp.256-281.

Grussenmeyer P., Alby E., Landes T., Koehl M., Guillemin S., Hullo J.F., Assali P., Smigiel E., 2012. Recording Approach of Heritage Sites based on Merging Point Clouds from High Resolution Photogrammetry and Terrestrial Laser Scanning. In: The International Archives of the Photogrammetry, Remote Sensing and Spatial Information Sciences Melbourne, Australia, Vol. XXXIX, Part B5, pp. $553-558$.

Markiewicz J, 2012. Photogrammetric data integration aspects for generation of 3D models of selected objects within the urban space. In: Archives of Photogrammetry, Cartography and Remote Sensing, Warsaw, Poland, vol. 24, pp 199- 209.

Moussa W., 2014. Integration of Digitall Photogrammetry and Terrstrial Laser Scanning for Cultural Heritage Data Recording. Verlag der Bayerischen Akademie der Wissenschaften in Kommission beim Verlag C.H.Beck, Studgart, Germany.

Ntegka A., Georgopoulos A., Santana Quintero M., 2013 Photogrammetric exploitation of HDR images for cultural heritage documentation. In: ISPRS Annals of the Photogrammetry, Remote Sensing and Spatial Information Sciences, Strasbourg, France Volume II-5/W1, pp.209 - 2014.
Remondino F., El-Hakim S., 2006. Image-based 3D modelling:a review. The Photogrammetric Record 21(115), pp. 269-291.

Remondino F., Menna F., 2008. Image-based surface measurement for close-range heritage documentation. In: The International Archives of the Photogrammetry, Remote Sensing and Spatial Information Sciences, Beijing, China, Vol. XXXVIII-5, Part B5, pp. 199 - 2006.

Lerma J. L., 2008. 3D RiskMapping. Theory and pratice on Terrestrial Laser Scanning. Tranning material based on pratical applications. http://jllerma.webs.upv.es/pdfs/ Leonardo_Tutorial_Final_vers5_ENGLISH.pdf (8.05.2014)

http://www.zf-laser.com/fileadmin/editor/Broschueren /Z_F_IMAGER_5010C_E_FINAL_kompr.pdf (10.05.2014). 